\title{
Análise Exploratória de Dados de Dosagens para modelos de previsão e estimativas de resistências à compressão de Concretos Autoadensáveis
}

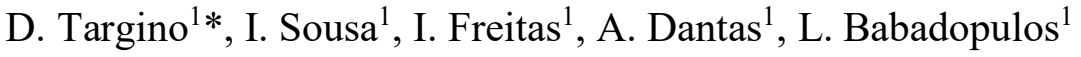 \\ *Autor de Contato: daniellira@alu.ufc.br \\ ${ }^{1}$ Departamento de Engenharia Estrutural e Construção Civil, Universidade Federal do Ceará, Fortaleza, Brasil.
}

\begin{abstract}
Machine learning models are capable of estimating strength parameters in concrete with high accuracy. For any of these, an initial exploratory analysis of the dataset is highly recommended, with investigation of their influence on the property or characteristic to be estimated, as well as their correlations. This study presents this analysis for a set of 505 concrete mixtures, containing consumption of raw materials, additives and additions, including specific masses of the basic material. As a result, in addition to the expected basic relationships, others not so explicit were evidenced, such as the strong influence of some specific masses on the compressive strength. Given the strong relationship of the characterization parameters, its need for the construction of more refined models, with application in different sources of inputs, is evidenced.
\end{abstract}

Key-words: exploratory data analysis; concrete mixtures; machine learning models; materials characterization.

\section{RESUMO}

Modelos de aprendizado de máquina possuem capacidade de estimar parâmetros de resistência em concretos com elevada precisão. Para qualquer destes é recomendado uma análise exploratória inicial dos dados de entrada, com investigação da influência desses na característica a ser estimada, bem como suas correlações. O presente estudo apresenta essa análise para um conjunto de 505 dosagens, contendo consumos de insumos básicos, aditivos e adições, além das massas específicas desses primeiros. Como resultado, além das relações básicas esperadas, outras não tão explícitas foram evidenciadas, como a forte influência de algumas massas específicas na resistência à compressão. Visto a forte relação dos parâmetros de caracterização, é evidenciada sua necessidade para a construção de modelos mais refinados, com aplicação em diferentes origens de insumos.

Palavras-Chave: análise exploratória de dados; dosagens de concretos; modelos de aprendizado; caracterização de materiais. 


\section{INTRODUÇÃO}

O concreto é o segundo material mais consumido no mundo (MONTEIRO; MILLER; HORVATH, 2017), com inúmeras adaptações na formação de seu compósito para atender às variadas demandas e aplicações desse material. O concreto convencional pode ser caracterizado pelos consumos de seus materiais, seja cimento, areia, brita e água, podendo também conter adições que contribuam para um desempenho superior (ABREU, 1998), resistindo principalmente a esforços de compressão.

$\mathrm{Na}$ tentativa de suprimir a necessidade de vibração do concreto após lançamento, foram $\mathrm{O}$ concreto é o segundo material mais consumido no mundo (MONTEIRO; MILLER; HORVATH, 2017), com diversas adaptações na formação de seu compósito para atender às variadas demandas e aplicações. O concreto convencional pode ser caracterizado pelos consumos de seus materiais, seja cimento, areia, brita e água, podendo também conter adições que contribuam para um desempenho superior (ABREU, 1998), resistindo principalmente a esforços de compressão.

Na tentativa de suprimir a necessidade de vibração do concreto após lançamento, foram desenvolvidas no Japão na década de 1980, as primeiras formulações de concreto autoadensável (BELALIA DOUMA et al., 2017), material este com capacidade de adensamento sobre o próprio peso, além de possuir em seu estado fresco elevada fluidez, habilidade passante entre ferragens e resistência à segregação (COSTA; CABRAL, 2019; KONERU; GHORPADE, 2019).

No processo de dosagem, a determinação dessas e outras características ocorre também por métodos de tentativa e erro (SHI et al., 2015), o que é responsável por processos mais demorados e maiores custos. Para o concreto autoadensável, esse passo é ainda mais complexo, em função de requisitos no estado fresco. Nesse panorama, gerar conhecimento a partir de dosagens prévias é uma das metodologias mais utilizadas, sendo que esse problema pode ser adaptado a estudos de outras áreas do conhecimento, como data science e machine learning da tecnologia da informação.

Inteligência artificial (IA) tem sido objeto de vários estudos e aplicações nos últimos anos, principalmente devido à sua potencialidade, desde detecção de fraudes em sistemas de pagamento (RYMAN-TUBB; KRAUSE; GARN, 2018; YAZICI, 2020) até diagnóstico de COVID-19 em imagens de raios-x (BORKOWSKI, 2020; SALMAN et al., 2020), onde para a implementação de algoritmos ou modelos de aprendizado, até mesmo outras metodologias estatísticas, é necessário o tratamento e análise dos dados a serem processados através da técnica de análise exploratória de dados (AED).

Para um bom entendimento do comportamento da amostra de dados é necessária a utilização de técnicas que permitam a visualização do conjunto de dados (dataset) sobre diferentes perspectivas, podendo ocorrer através da análise descritiva da base de dados, análise gráfica, por meio de diagramas, histogramas ou outros gráficos pertinentes e metodologias específicas de análises de correlações, como a metodologia da correlação de Pearson, sendo que o intuito é de demonstrar correlações e dependências não percebidas inicialmente.

Dessa forma, o presente estudo se propõe a realizar uma AED em um banco de dados de 505 instâncias de dosagens de concretos autoadensáveis, onde cada instância possui atributos relativos a consumos de materiais básicos, aditivos e adições, quando utilizados, juntamente aos 
dados de caracterização de massa específica dos materiais básicos. Dados de consumos são mais comuns em processos de implementação de modelos de aprendizados para dosagens de concretos, nesse caso pretende-se particularmente avaliar a correlação entre os parâmetros de caracterização dos materiais com a resistência à compressão, juntamente às análises cruzadas entre os mesmos. Essa análise e organização de dados é uma contribuição deste trabalho que será continuada em trabalhos futuros, com implementações de diferentes IA e a avaliação de suas performances.

\section{METODOLOGIA}

A metodologia é dividida de tal forma em que cada seção abordará um processo específico da metodologia geral apresentada na Figura 01.

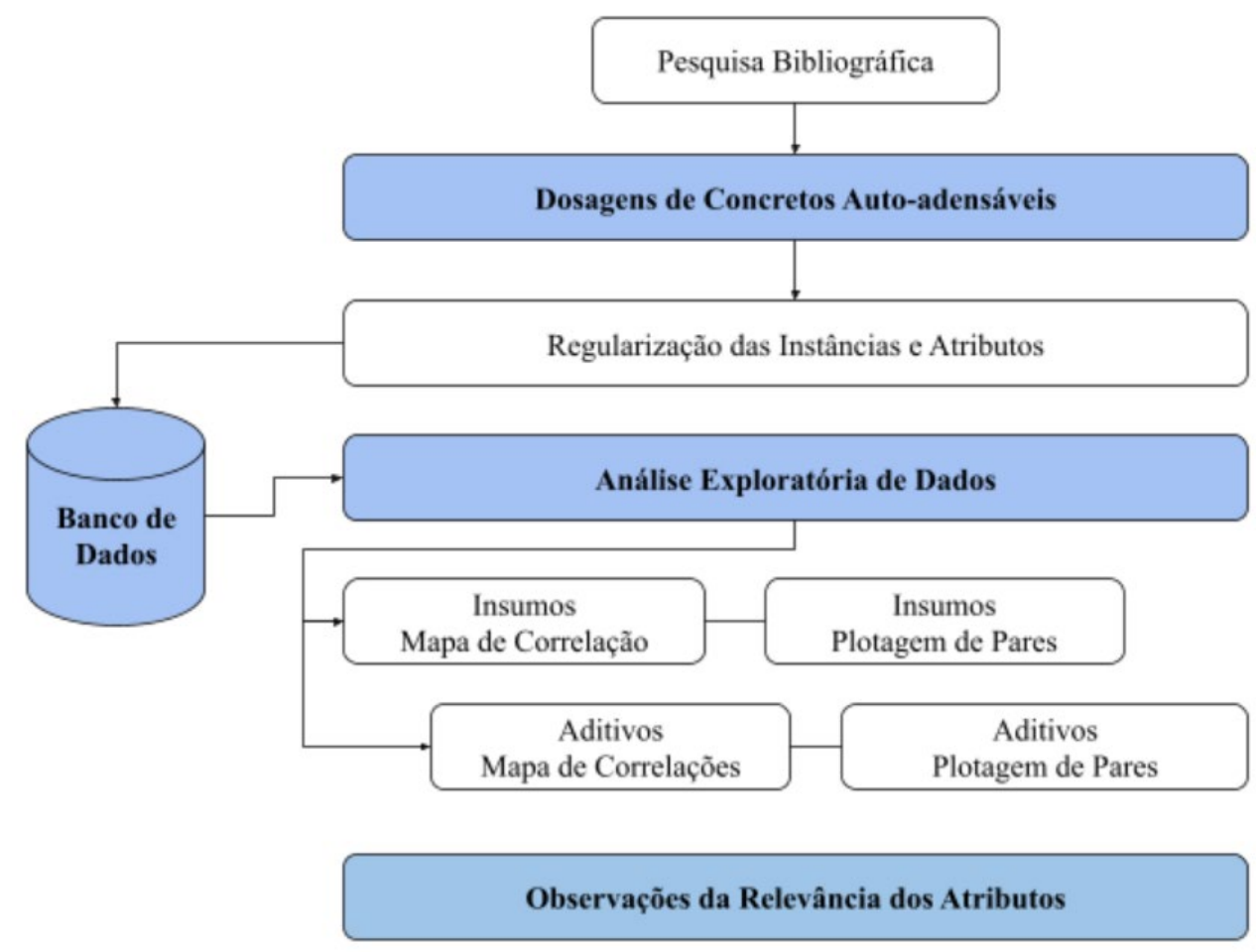

Figura 01. Fluxograma da Metodologia. Autor, 2021.

\subsection{Banco de Dados, Atributos das Instâncias e Variáveis Resultados}

A coleta de dosagens para o banco de dados foi feita por meio da pesquisa bibliográfica em artigos científicos e dissertações, nacionais e internacionais sobre concretos autoadensáveis (CAA). Por cada instância entenda-se como uma dosagem de concreto, com os dados de consumo de cimento, areia, brita, água, aditivos e adições. O resumo dos artigos utilizados é apresentado na Tabela 1. 
Tabela 1. Resumos bibliografias utilizadas no banco de dados. Autor, 2021.

\begin{tabular}{|c|c|c|}
\hline Referência & Número de Instâncias & Autor \\
\hline 1 & 123 & (KONERU; GHORPADE, 2019) \\
\hline 2 & 28 & (TUTIKIAN, 2004) \\
\hline 3 & 8 & (BENAICHA et al., 2019) \\
\hline 4 & 69 & (ABU YAMAN; ABD ELATY; TAMAN, 2017) \\
\hline 5 & 114 & (BELALIA DOUMA et al., 2017) \\
\hline 6 & 15 & (BASU; GUPTA; AGRAWAL, 2020) \\
\hline 7 & 12 & (FERREIRA; RIBEIRO, 2018) \\
\hline 8 & 3 & (ALMEIDA FILHO et al., 2008) \\
\hline 9 & 3 & (ALESSANDRO ALMEIDA DINIZ et al., 2018) \\
\hline 10 & 65 & (GÜNEYISI; GESOLU; ÖZBAY, 2010) \\
\hline 11 & 12 & (JALAL; MANSOURI, 2012) \\
\hline 12 & 10 & (RAMANATHAN et al., 2013) \\
\hline 13 & 10 & (SUKUMAR; NAGAMANI; SRINIVASA RAGHAVAN, 2008) \\
\hline 14 & 10 & (BOUZOUBAÂ; LACHEMI, 2001) \\
\hline 15 & 9 & (BOEL et al., 2007) \\
\hline 16 & 7 & (NEHDI; PARDHAN; KOSHOWSKI, 2004) \\
\hline 17 & 7 & (BINGÖL; TOHUMCU, 2013) \\
\hline Total & 505 & \\
\hline
\end{tabular}

Os dados de entradas foram divididos em três categorias principais: consumo de materiais básicos (cimento, areia, brita e água), consumo de aditivos e adições (sílica ativa, plastificantes, dentre outros) e os dados de caracterização das resistências (compressão axial, tração, flexão e módulo de elasticidade). Foi necessário um tratamento inicial dos dados devido à alta heterogeneidade de apresentação destes, visto que é usual que consumos sejam apresentados como fator ou como percentual em relação à massa de outro material. Dessa forma, neste processo, todas as instâncias foram convertidas para unidades de consumo de material em massa por volume de concreto, bem como suas unidades de massa específica.

Ao final, o banco de dados utilizado compreendeu um total de 505 instâncias de dosagens de concretos auto adensáveis, com 18 atributos cada, características de consumo de materiais e massas específicas, sem valores faltantes, medidos em unidade de quilograma por metro cúbico $\left(\mathrm{kg} / \mathrm{m}^{3}\right)$, resistências em megapascal $(\mathrm{MPa})$ 


\subsection{Análise Exploratória Inicial}

Nesta etapa pretende-se investigar correlações entre parâmetros não percebidos inicialmente no dataset. Essa metodologia possui alguns gráficos para facilitar o processo de verificação dessas correlações, como ilustrações de mapa de calor (heatmap), plotagem de pares cruzadas (pairplots), gráficos de pontos ponderados (weighted scatterplot), dentre outros. Cada um desses possui a capacidade de demonstrar tendências específicas, em relação a uma fatia específica do dataset, não percebidas inicialmente. Correlações positivas indicam relação direta, consequentemente, as negativas relações inversas ou destrutivas.

Nas correlações de Pearson (heatmap) essa dinâmica é facilmente visualizada, através dos coeficientes gerados, já nos pairplots é possível observar a distribuição e disposições entre os diferentes atributos, enquanto no weighted scatterplot é possível correlacionar, agrupar e distribuir mais de três dimensões de variáveis, com visualização e comportamento e agrupamento.

\section{RESULTADOS E DISCUSSÕES}

A Figura 02 apresenta o mapa de calor para atributos dos materiais básicos e resistência à compressão. O mesmo utiliza a correlação de Pearson, implementada na biblioteca Seaborn para Python. Os valores estão normalizados, variando entre $-0,68$ a 0,67 , adimensional.

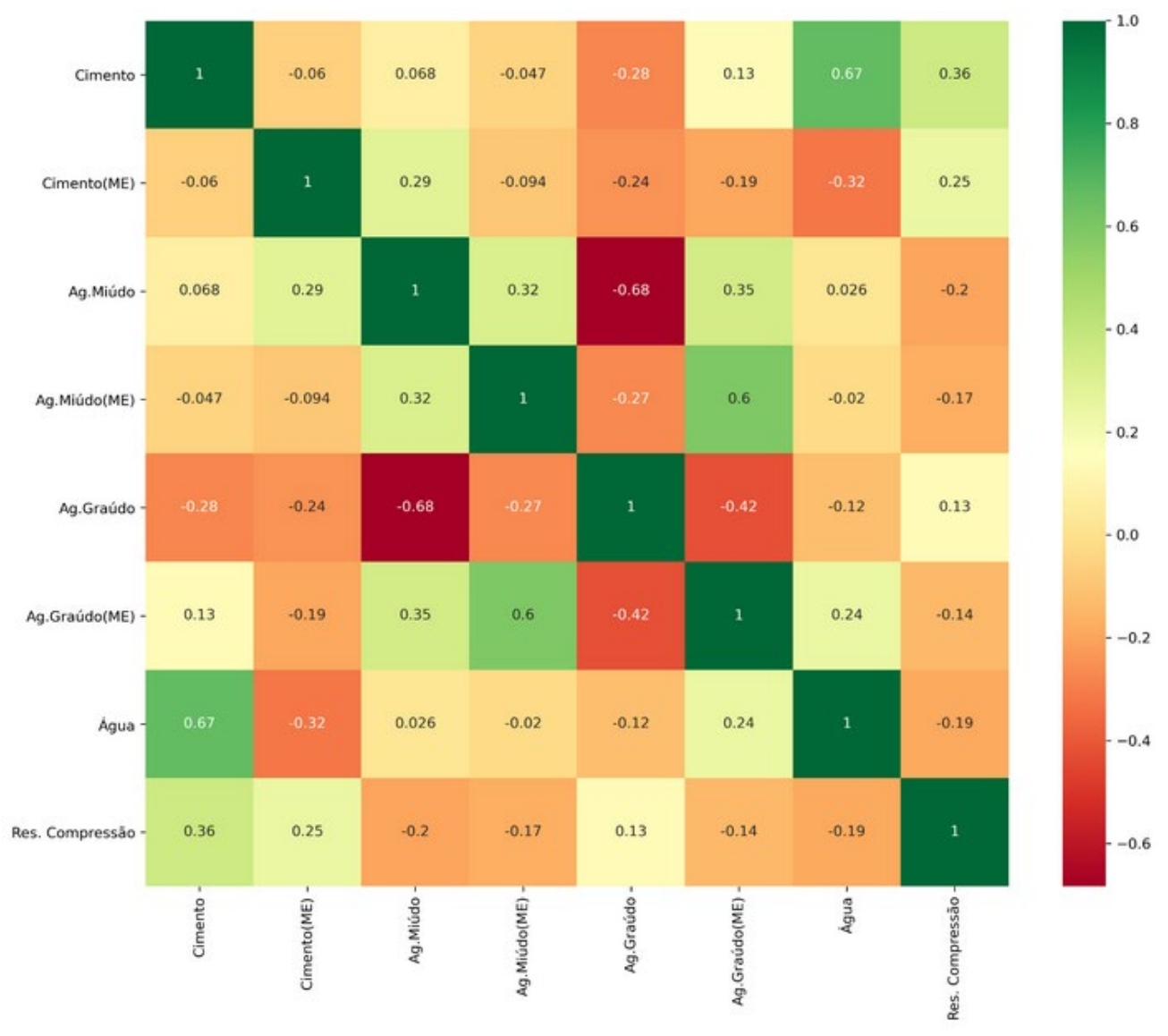


Figura 02. Heatmap de correlação entre atributos de materiais básicos e resistência. Autor, 2021.

Analisando as influências dos atributos na resistência à compressão, é possível observar a correlação positiva do consumo de cimento $(0,36)$, seguido por sua respectiva massa específica $(0,25)$, sendo também influenciada pelo consumo do agregado graúdo $(0,13)$. A influência da massa específica do cimento Portland é um aspecto não tão abordado em processos de dosagem. Pelas correlações observadas é possível inferir que cimentos de maior massa específicos terão maior contribuição para a resistência. A respeito do consumo de agregado graúdo, a análise evidencia forte relação frente aos demais insumos, o que possui bom embasamento teórico, tendo em vista que ocupa um volume elevado dentro do compósito e possui elevada resistência à compressão.

Os demais componentes apresentaram correlação negativa, valendo ressaltar que tanto o consumo de agregado miúdo quanto o consumo de água apresentaram correlações negativas da ordem de -0,20. A relação do consumo de água é algo já esperado, tendo em vista a influência do fator água-cimento, contudo relativo ao consumo do agregado miúdo é outro ponto a se ressaltar, pois tanto seu consumo quanto o aumento de sua massa específica (correlação de -0,17) possuem correlação negativa com o aumento da resistência.

Analisando as correlações entre os atributos é perceptível que a maior correlação positiva ocorre entre os consumos de água e cimento $(0,67)$, o que pode ser explicado pelo aumento da demanda de água necessária à hidratação do cimento, onde ambos valores estão diretamente relacionados à resistência final. Em segundo é observado as maiores relações das massas específicas dos agregados miúdo e graúdos $(0,60)$, explicado pelas relações de densidade. Em contrapartida, a maior correlação negativa ocorre em relação aos consumos desses últimos insumos em si $(-0,68)$, explicado pela tendência de preenchimento dos volumes internos de ambos os materiais, e relação entre a massa específica e consumo do graúdo $(-0,42)$.

A Figura 03 apresenta o mapa de calor para atributos dos aditivos, adições e resistência à compressão. Os coeficientes estão normalizados, variando entre -0,25 a 0,39. 


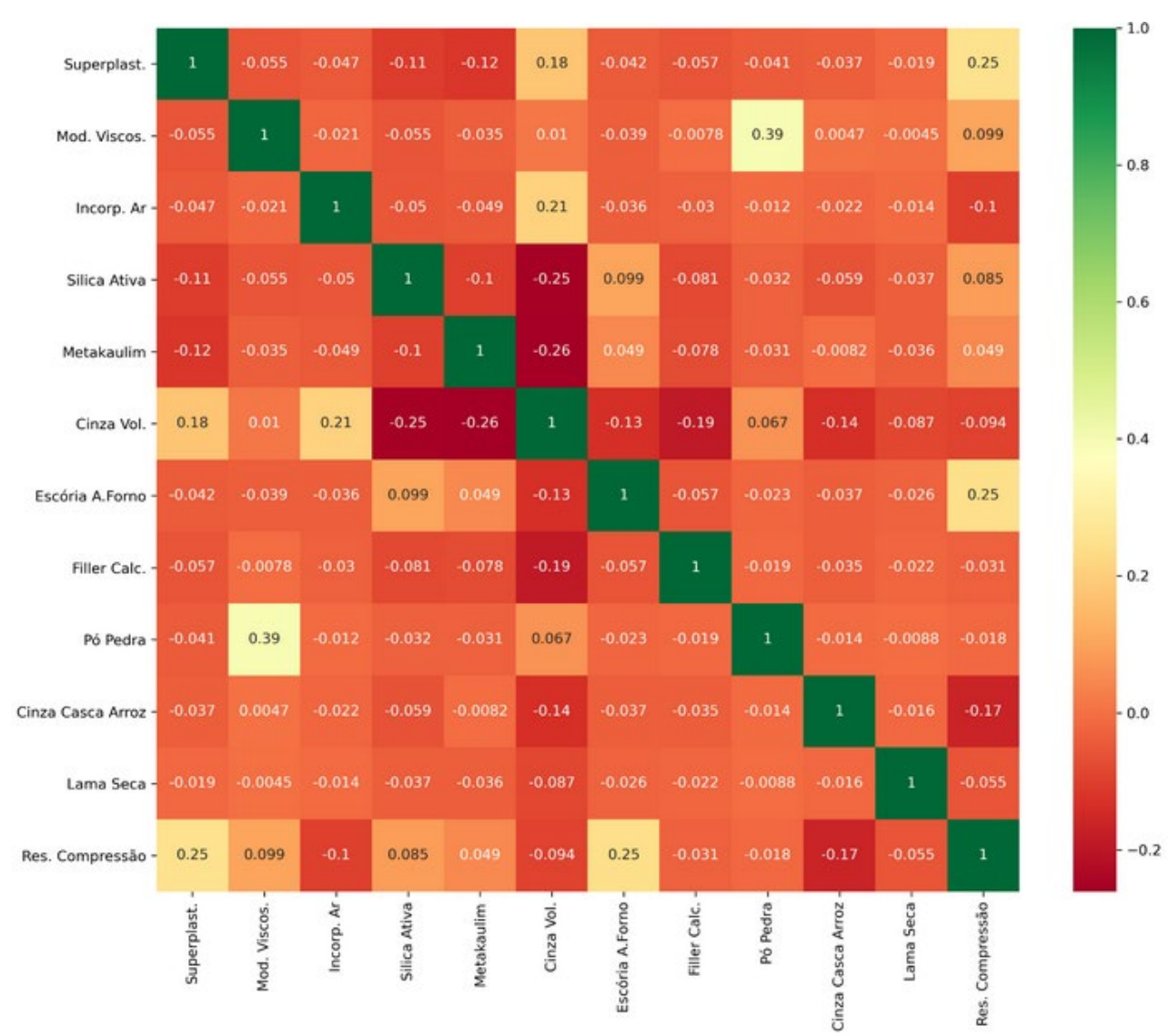

Figura 03. Heatmap de correlação entre atributos de aditivos, adições e resistência. Autor, 2021.

Analisando as influências na resistência à compressão, é possível observar a maior correlação positiva do consumo de superplastificante $(0,25)$, juntamente ao consumo de escória de alto forno $(0,25)$. O consumo de superplastificante permite reduzir o fator água-cimento contribuindo para a trabalhabilidade no estado fresco, influenciando diretamente a resistência. De forma similar, a escória de alto-forno possui propriedades pozolânicas, logo sua adição ao compósito deverá contribuir para o incremento da resistência. Os atributos do modificador de viscosidade, sílica ativa e metacaulim obtiveram correlações menores, porém com contribuições positivas, enquanto todos os demais foram negativos, em especial a cinza de casca de arroz com a contribuição mais negativa $(-0,17)$.

Analisando as correlações entre os atributos é perceptível que a maior correlação positiva ocorre entre os consumos de pó de pedra e modificador de viscosidade $(0,39)$, seguido pelo consumo de cinza volante e incorporador de ar $(0,21)$ e superplastificante $(0,18)$. Em geral, as relações acima evidenciam interferências direta entre consumos, onde o aumento do consumo das adições (pó de pedra e cinza volante) irão demandar o aumento do consumo desses aditivos em específico (modificador de viscosidade, incorporador de ar e superplastificante)

Em contrapartida, a maior correlação negativa ocorre em relação ao consumo da cinza em comparação à sílica ativa $(-0,68)$, o que pode ser explicado pela similaridade de função que ambos possuem, de maneira que o aumento de consumo de uma parte deverá reduzir o outro consumo. 


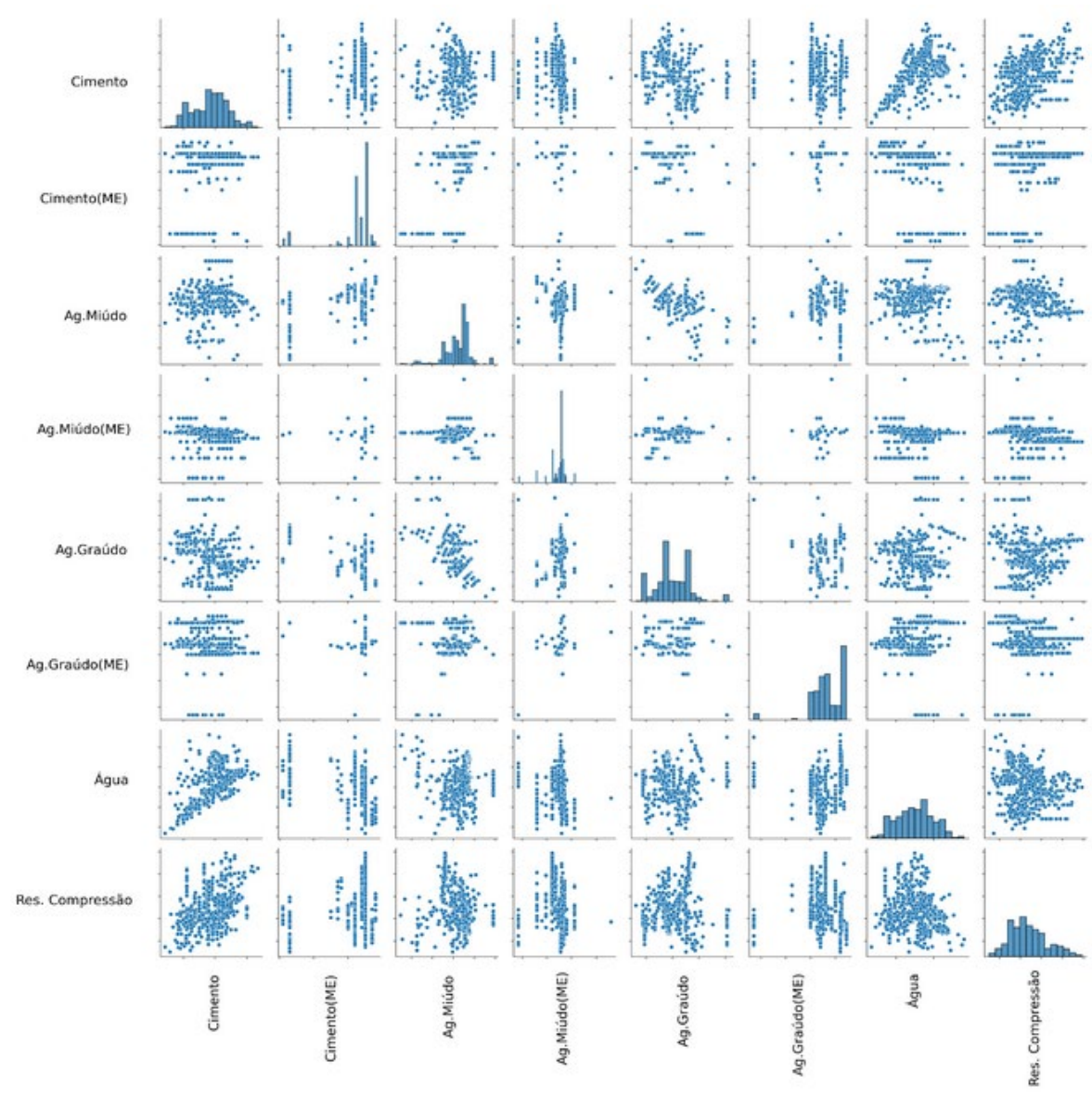

Figura 04. Pairplot de correlação entre materiais básicos e resistência. Autor, 2021.

A Figura 04 apresenta o pairplot com a correlação entre os materiais básicos e a resistência à compressão. Os gráficos apresentam as combinações dois a dois de cada atributo em acréscimo à variável de saída.

O comportamento observado dos dados é bastante heterogêneo. Na diagonal principal é apresentada as distribuições de dados dos atributos. Os pares consumo de cimento versus consumo de água e resistência compressão apresentaram distribuições próximas à uma tendência linear, indicando boa correlação. Analisando a distribuição dos dados de massa específica dos agregados é percebido que existe uma alta concentração entre diferentes dosagens, evidenciando uma similaridade dos processos de formação e obtenção desses materiais. O mesmo é observado na caracterização de massa específica dos cimentos utilizados. 
A Figura 05 evidencia o pairplot com a correlação entre os aditivos, adições e resistência à compressão. Os valores estão normalizados e mostram o comportamento dos dados dispostos no gráfico.

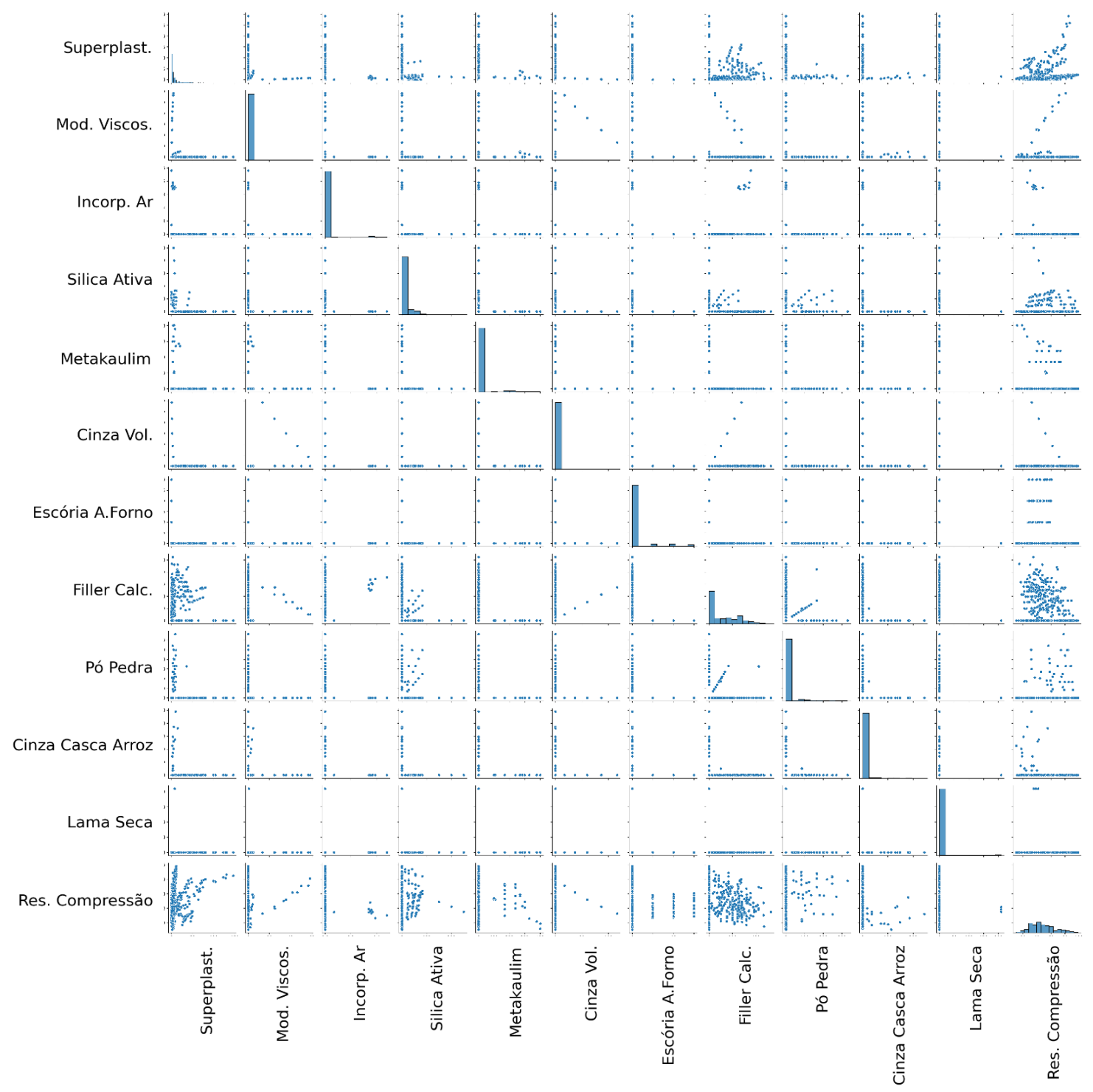

Figura 05. Pairplot de correlação entre atributos de aditivos, adições e resistência. Autor, 2021. 
O segundo pairplot apresenta a heterogeneidade similar ao primeiro gráfico, com comportamentos mais singulares. Em linhas gerais é possível perceber que as dosagens do banco de dados analisado tendem a abordar apenas um parâmetro de aditivo ou adição por grupo de dosagem. Essa tendência é observada pela alta concentração de dados próximos aos eixos x e y. Os gráficos que obtiveram maiores dispersões ao longo dos eixos foram os relativos aos aditivos mais comumente utilizados, como superplastificantes, sílica ativa e filler calcário, o que indica a presença desses em conjunto à análise de outras adições na literatura e dataset analisados

A Figura 06 indica uma correlação entre diferentes insumos do concreto auto adensável e a resistência à compressão.

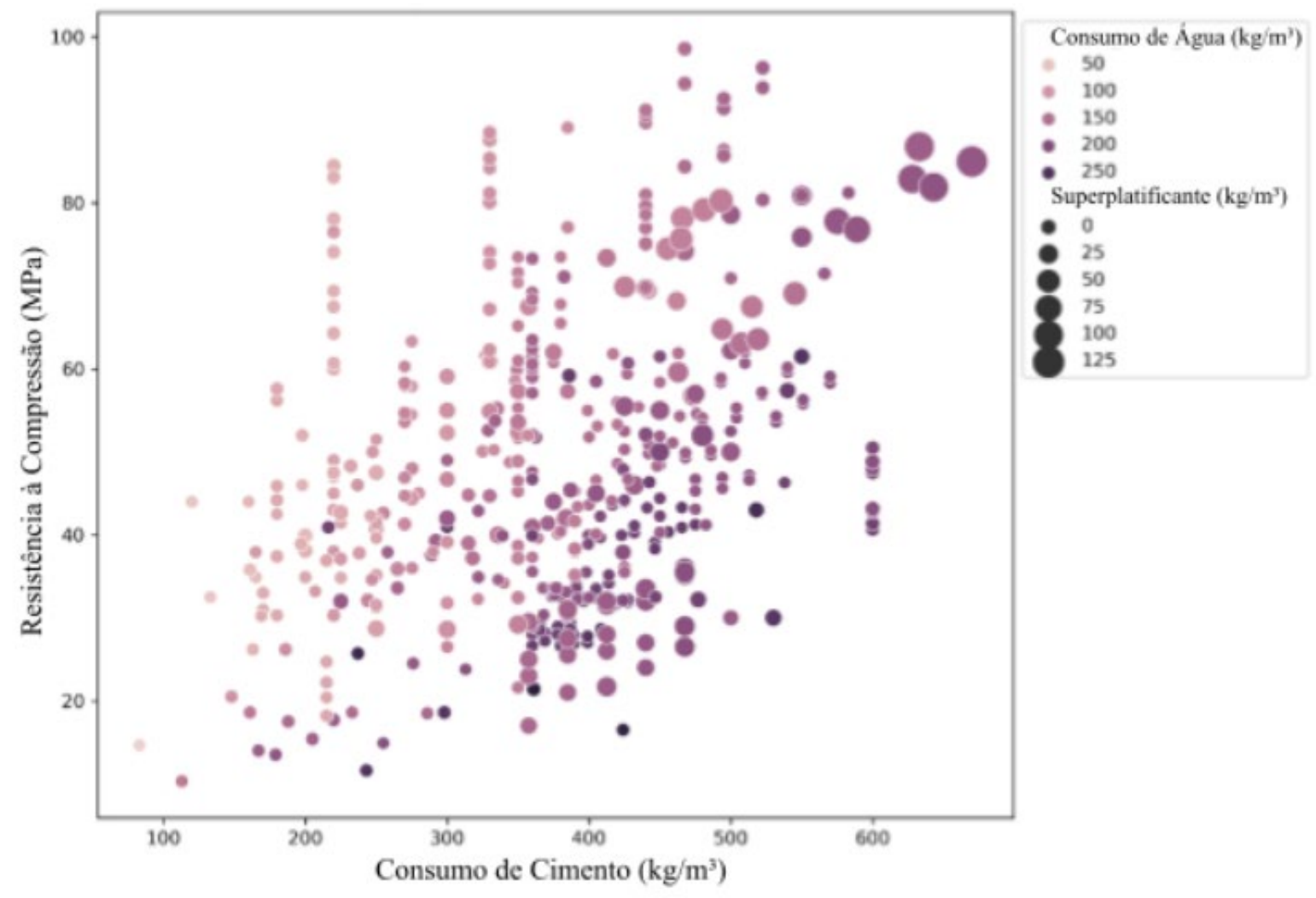

Figura 06. Consumo de cimento, água, plastificante versus resistência à compressão. Autor, 2021.

Na Figura 06, percebe-se que à medida que o ocorre o aumento do consumo de cimento, ocorre também uma tendência ao aumento da resistência à compressão, onde a maior densidade de dados de alta resistência (acima de $50 \mathrm{MPa}$ ) está localizada entre os intervalos de $400 \mathrm{~kg} / \mathrm{m}^{3}$ e 500 $\mathrm{kg} / \mathrm{m}^{3}$ de consumo desse primeiro.

Da mesma forma, é evidenciada a relação entre o consumo de água e a resistência, indicando uma tendência à obtenção de maiores valores de resistência relacionados ao consumo baixo e intermediário de água, entre $50 \mathrm{~kg} / \mathrm{m}^{3}$ e $150 \mathrm{~kg} / \mathrm{m}^{3}$, ao passo que para maiores valores do seu consumo, observa-se uma menor resistência. 
Já quanto à utilização de superplastificante, observa-se uma tendência à maior utilização desse aditivo para maiores quantidades de consumo de cimento, como observado nos valores superiores a $600 \mathrm{~kg} / \mathrm{m}^{3}$ e na faixa de $450 \mathrm{~kg} / \mathrm{m}^{3}$ de consumo desse insumo.

A resistência à compressão dos concretos dispostos no banco de dados tem sua curva de distribuição apresentada na Figura 07.

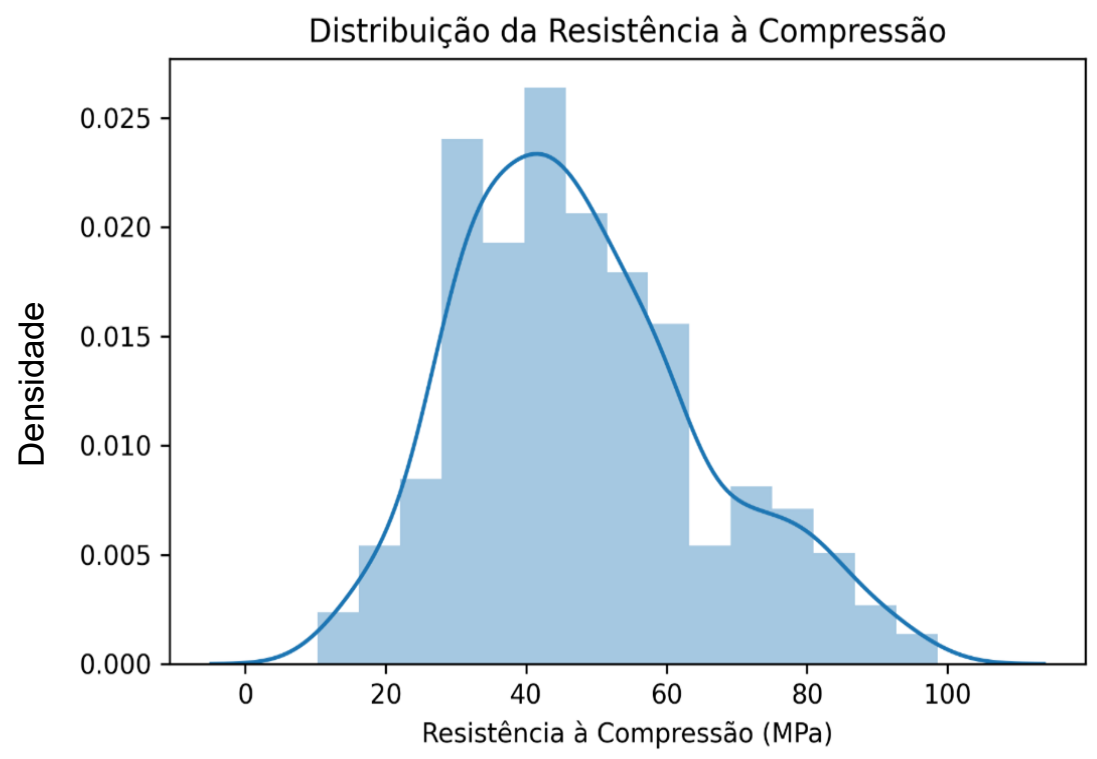

Figura 07. Curva de distribuição da resistência à compressão do banco de dados. Autor, 2021.

Portanto, percebe-se através da curva de distribuição, uma tendência à obtenção de concretos com a resistência média de 47,92, com desvio padrão de 17,51, variando de 10,30 à 98,60 $\mathrm{MPa}$. O total de instâncias com resistência superior a $50 \mathrm{MPa}$ possuem uma menor densidade de amostras distribuídas no banco de dados, sendo as maiores incidências nas faixas de 35 e $45 \mathrm{MPa}$.

\section{CONCLUSÕES}

A análise estatística exploratória inicial correlacionou os dados que possuem sentido físico nas propriedades observados no processo de dosagem (Figuras 02 e 03), como a influência do fator água-cimento na resistência, relação de consumo dos agregados, aumento na resistência por influência dos materiais pozolânicos, dentre outros.

A análise de dados acima demonstra uma forte relação não só dos consumos, como também das caracterizações dos materiais utilizados. Esse dado se faz relevante a processos de análises e modelos de aprendizado devido à alta variabilidade de materiais que podem ser empregados na elaboração de compósitos estruturais.

A exemplo, é possível perceber, pelas análises aqui executadas, a influência da massa específica do cimento na resistência à compressão, juntamente à influência positiva do consumo dos agregados graúdos, cogitando a possibilidade de formulações de dosagens maximizando esses dois atributos em específico. 
Sobre a ótica dos aditivos e adições, é possível perceber a influência cruzada apenas dos itens mais básicos (Superplastificantes, Modificadores e Sílica). Nas análises bibliográficas utilizadas foi verificado que cada artigo ou bibliografia se dedica basicamente à um ou outro item de forma isolada, o que é algo positivo para investigação da influência do item investigado, contudo, deixando aquém a correlação entre diferentes aditivos e adições.

Os métodos de dosagens de concretos atuais, ainda são dependentes de validações e testes em laboratório, com os respectivos ajustes. Com base nas correlações aqui dispostas já é possível estimar influências de parâmetros para os ajustes requeridos, servindo de base também para a implementação de modelos de aprendizados ou redes neurais para a previsão de propriedades. As Tabela 2 e 3 apresenta um resumo das principais correlações dos atributos em relação à resistência à compressão.

Tabela 2. Resumos de contribuições dos materiais básicos na resistência. Autor, 2021.

\begin{tabular}{|c|c|c|}
\hline Item & Coeficiente Estatístico & Atributo de Dosagem com Influência na Resistência \\
\hline 1 & 0,36 & Consumo de Cimento \\
\hline 2 & 0,25 & Massa Específica do Cimento \\
\hline 3 & 0,13 & Consumo de Agregado Graúdo \\
\hline 4 & $(-0,17)$ & Massa Específica do Agregado Miúdo \\
\hline 5 & $(-0,19)$ & Consumo de Água \\
\hline 6 & $(-0,20)$ & Consumo de Agregado Miúdo \\
\hline
\end{tabular}

Tabela 3. Resumos de contribuições dos aditivos e adições na resistência. Autor, 2021.

\begin{tabular}{|c|c|c|}
\hline Item & Coeficiente Estatístico & Atributo de Dosagem com Influência na Resistência \\
\hline 1 & 0,25 & Consumo Superplastificante e Escória de Alto Forno \\
\hline 2 & 0,099 & Consumo de Modificador de Viscosidade \\
\hline 3 & 0,085 & Consumo de Sílica Ativa \\
\hline 4 & $(-0,094)$ & Consumo de Cinza Volante \\
\hline 5 & $(-0,10)$ & Consumo de Incorporador de Ar \\
\hline 6 & $(-0,17)$ & Consumo da Cinza da Casca de Arroz \\
\hline
\end{tabular}

As Tabelas 4 e 5 apresentam um resumo das correlações entre atributos em geral para os insumos básicos (Tabela 4) e aditivos e adições (Tabela 5). 
Tabela 4. Resumos de correlações entre atributos de materiais básicos. Autor, 2021.

\begin{tabular}{|c|c|c|}
\hline Item & Coeficiente Estatístico & Correlações entre Atributos \\
\hline 1 & 0,67 & Consumo de Água vs. Consumo de Cimento \\
\hline 2 & 0,60 & M. E. Agregado Graúdo vs. M. E. Agregado Miúdo \\
\hline 3 & 0,35 & M. E. Agregado Graúdo vs. Consumo de Agregado Miúdo \\
\hline 4 & $(-0,32)$ & Consumo de Água vs. M. E. Cimento \\
\hline 5 & $(-0,42)$ & M. E. Agregado Graúdo vs. Consumo de Agregado Graúdo \\
\hline 6 & $(-0,68)$ & Consumo de Agregado Graúdo vs. Consumo de Agregado Miúdo \\
\hline
\end{tabular}

Tabela 5. Resumos de correlações entre atributos de aditivos e adições. Autor, 2021.

\begin{tabular}{|c|c|c|}
\hline Item & Coeficiente Estatístico & Correlações entre Atributos (Consumos) \\
\hline 1 & 0,39 & Pó de Pedra vs. Modificador de Viscosidade \\
\hline 2 & 0,21 & Cinza Volante vs. Incorporador de Ar \\
\hline 3 & 0,18 & Cinza Volante vs. Superplastificante \\
\hline 4 & $(-0,19)$ & Filler Calcário vs. Cinza Volante \\
\hline 5 & $(-0,25)$ & Cinza Volante vs. Sílica Ativa \\
\hline 6 & $(-0,26)$ & Cinza Volante vs. Metacaulim \\
\hline
\end{tabular}

\section{AGRADECIMENTOS}

O presente trabalho foi realizado com apoio do Conselho Nacional de Desenvolvimento Científico e Tecnológico (CNPq) e da Fundação Cearense de Apoio ao Desenvolvimento Científico e Tecnológico (Funcap).

\section{BIBLIOGRAFIA}

ABREU, A. G. DE. Efeito das adições minerais na resistividade elétrica de concretos convencionais. Dissertação (Mestrado em Engenharia Civil) - Escola de Engenharia da Universidade Federal do Rio Grande do Sul. Porto Alegre, p. 142. 1998.

ABU YAMAN, M.; ABD ELATY, M.; TAMAN, M. Predicting the ingredients of self-compacting concrete using artificial neural network. Alexandria Engineering Journal, v. 56, n. 4, p. 523$532,2017$. 
ALESSANDRO ALMEIDA DINIZ, H. et al. Caracterização E Avaliação Das Propriedades De Concretos Autoadensáveis Com Adições Minerais E Baixo Consumo De Cimento. Holos, v. 5, p. 51-64, 2018.

ALMEIDA FILHO, F. M. et al. Variabilidade da aderência e das propriedades mecânicas do concreto autoadensável. Revista IBRACON de Estruturas e Materiais, v. 1, n. 1, p. 31-57, 2008.

BASU, P.; GUPTA, R. C.; AGRAWAL, V. Effects of sandstone slurry, the dosage of superplasticizer and water/binder ratio on the fresh properties and compressive strength of selfcompacting concrete. Materials Today: Proceedings, v. 21, p. 1250-1254, 2020.

BELALIA DOUMA, O. et al. Prediction of properties of self-compacting concrete containing fly ash using artificial neural network. Neural Computing and Applications, v. 28, n. s1, p. 707718, 2017.

BENAICHA, M. et al. Dosage effect of superplasticizer on self-compacting concrete: Correlation between rheology and strength. Journal of Materials Research and Technology, v. 8, n. 2, p. 2063-2069, 2019.

BINGÖL, A. F.; TOHUMCU, I. Effects of different curing regimes on the compressive strength properties of self-compacting concrete incorporating fly ash and silica fume. Materials and Design, v. 51, p. 12-18, 2013.

BOEL, V. et al. Transport properties of self-compacting concrete with limestone filler or fly ash. Materials and Structures/Materiaux et Constructions, v. 40, n. 5, p. 507-516, 2007.

BORKOWSKI, A. Using Artificial Intelligence for COVID-19 Chest X-ray Diagnosis. Federal Practitioner, v. 19, n. Vol 37 No 9, p. 398-404, 2020.

BOUZOUBAÂ, N.; LACHEMI, M. Self-compacting concrete incorporating high volumes of class F fly ash: Preliminary results. Cement and Concrete Research, v. 31, n. 3, p. 413-420, 2001.

COSTA, A. C. S. DE S.; CABRAL, A. E. B. Estudo comparativo entre o concreto autoadensável e o concreto convencional vibrado em obra vertical. Ambiente Construído, v. 19, n. 4, p. 289$301,2019$.

FERREIRA, L.; RIBEIRO, F. Estudo Comparativo do Concreto Auto Adensável com Cinza da Casca de Arroz sem Controle de Queima e Sílica Ativa. Paranoá: cadernos de arquitetura e urbanismo, n. 20, p. 1-12, 2018.

GÜNEYISI, E.; GESOLU, M.; ÖZBAY, E. Strength and drying shrinkage properties of selfcompacting concretes incorporating multi-system blended mineral admixtures. Construction and Building Materials, v. 24, n. 10, p. 1878-1887, 2010.

JALAL, M.; MANSOURI, E. Effects of fly ash and cement content on rheological, mechanical, and transport properties of high-performance self-compacting concrete. Science and Engineering of Composite Materials, v. 19, n. 4, p. 393-405, 2012. 
KONERU, V. S.; GHORPADE, V. G. Assessment of strength characteristics for experimental based workable self-compacting concrete using artificial neural network. Materials Today: Proceedings, v. 26, n. xxxx, p. 1238-1244, 2019.

MONTEIRO, P. J. M.; MILLER, S. A.; HORVATH, A. Towards sustainable concrete. Nature Materials, v. 16, n. 7, p. 698-699, 2017.

NEHDI, M.; PARDHAN, M.; KOSHOWSKI, S. Durability of self-consolidating concrete incorporating high-volume replacement composite cements. Cement and Concrete Research, v. 34, n. 11, p. 2103-2112, 2004.

RAMANATHAN, P. et al. Performance of self-compacting concrete containing different mineral admixtures. KSCE Journal of Civil Engineering, v. 17, n. 2, p. 465-472, 2013.

RYMAN-TUBB, N. F.; KRAUSE, P.; GARN, W. How Artificial Intelligence and machine learning research impacts payment card fraud detection: A survey and industry benchmark. Engineering Applications of Artificial Intelligence, v. 76, n. June, p. 130-157, 2018.

SALMAN, F. M. et al. COVID-19 Detection using Artificial Intelligence. International Journal of Academic Engineering Research, v. 4, n. 3, p. 18-25, 2020.

SHI, C. et al. A review on mixture design methods for self-compacting concrete. Construction and Building Materials, v. 84, p. 387-398, 2015.

SUKUMAR, B.; NAGAMANI, K.; SRINIVASA RAGHAVAN, R. Evaluation of strength at early ages of self-compacting concrete with high volume fly ash. Construction and Building Materials, v. 22, n. 7, p. 1394-1401, 2008.

TUTIKIAN, B. F. Método para dosagem de concreto autoadensável. Dissertação (Mestrado em Engenharia Civil) - Universidade Federal do Rio Grande do Sul. Porto Alegre, p. 149. 2004.

YAZICI, Y. Approaches to Fraud Detection on Credit Card Transactions using Artificial Intelligence Methods. Machine Learning, p. 235-244, 2020. 